\title{
激光产生的硅等离子体辐射动力学特性研究
}

\author{
敏琦，苏茂根"，曹世权，孙对兄，董晨钟" \\ 西北师范大学物理与电子工程学院, 甘肃省原子分子物理与功能材料重点实验室, 兰州 730070 \\ *联系人, 董晨钟, E-mail: dongcz@nwnu.edu.cn; 苏茂根, E-mail: sumg@nwnu.edu.cn
}

收稿日期: 2016-01-19; 接受日期: 2016-03-15; 网络出版日期: 2016-07-08

国家自然科学基金(编号: 11274254, U1332206, 11064012, 11364037, 11564037)、甘肃省高等学校基本科研业务费项目和甘肃省自然科 学基金(编号: 1308RJZA166, 1104WCGA186)资助

\begin{abstract}
摘要 本文从实验和理论两方面系统研究了真空环境中激光产生的硅等离子体的辐射动力学特性. 实验上利 用时间-空间分辨的激光等离子体发射光谱技术, 测量了激光产生的硅等离子体极真空紫外波段的光谱, 并对光 谱进行了分析指认. 为了进一步研究实验光谱的时空演化特性, 提出了一种简化的基于流体动力学和辐射输运 方程的等离子体辐射动力学模型, 利用该模型研究了激光产生的等离子体的辐射特性和动力学演化行为, 并对 实验观测到的时空分辨光谱进行了模拟, 详细分析了等离子体的温度、密度和离子布居的时空演化特性. 研究结 果表明, 本文提出的模型能够较好地反映激光等离子体的动力学演化过程以及辐射光谱的特征, 可以作为一种 有效的激光等离子体诊断工具.
\end{abstract}

关键词 激光产生的 $\mathrm{Si}$ 等离子体, 时空分辨光谱, 辐射动力学模型

PACS: $32.30 . J c, 39.30 .+w, 47.10 . a b, 57.70 . \mathrm{Kz}$

当一束高能激光脉冲聚焦于真空中的固体样品 表面时会烧蚀产生高温高密的等离子体. 等离子体 在膨胀冷却过程中会涉及大量微观粒子(原子、离子、 电子等)间复杂的相互碰撞和作用过程，同时伴随着 频率范围很宽的光发射. 因此, 激光等离子体瞬态图 像和光谱的实验观测以及理论研究可用来揭示大量 有关原子(离子)的能级结构, 以及等离子体状态的演 化信息. 激光等离子体光谱技术也是目前开展惯性 约束聚变、磁约束聚变、天体等离子体诊断和 $\mathrm{X}$ 射 线激光等领域研究的重要工具之一 ${ }^{[1]}$.
在激光等离子体中各种辐射过程与粒子的运动 和能量输运紧密相关, 光谱结构比较复杂, 难以进行 快速准确的分析研究. 特别对于激光烧蚀中 $Z$ 或高 $Z$ 元素产生的高电荷态离子, 谱线数目繁多、多种电荷 态离子光谱重叠等问题导致其光谱结构一般呈现较 宽的谱线轮廓或准连续特征. 同时由于等离子体不 透明度效应的影响, 使得等离子体的状态诊断和光 谱结构分析变得很困难. 因此, 对等离子体光谱结构 和辐射诊断研究不仅需要高分辨的光谱测量技术, 同时还需要精确的等离子体离子布居动力学计算.

引用格式: 敏琦, 苏茂根, 曹世权, 等. 激光产生的硅等离子体辐射动力学特性研究. 中国科学: 物理学 力学 天文学, 2016, 46: 083011 Min Q, Su M G, Cao S Q, et al. Investigation of radiation dynamic properties in laser-produced silicon plasma (in Chinese). Sci Sin-Phys Mech Astron, 2016, 46: 083011, doi: 10.1360/SSPMA2016-00032 
近年来, 国内外许多研究小组已经开展了有关 激光等离子体动力学性质的研究工作. 在实验方面, 美国 Lawrence Livermore 国家实验室的 Glenzer 等人 ${ }^{[2]}$ 测量了惯性约束聚变(ICF)黑腔中金等离子体的离 子价态分布. 北京应用物理与计算数学所的 $\mathrm{Xu}$ 等人 ${ }^{[3]}$ 利用神光 II 激光装置实现了时间分辨的 $\mathrm{Al}$ 等离子 体吸收谱测量. 美国的 Harilal 等人 ${ }^{[4]}$ 利用门宽为 $2 \mathrm{~ns}$ 的 ICCD 相机拍摄了环境气体压强在 $1 \times 10^{-6}-100$ $\operatorname{Torr}(1 \mathrm{Torr}=133.3 \mathrm{~Pa})$ 范围的激光铝等离子体羽演化 图像，获得了等离子体尺寸随时间的演化信息. 该小 组还利用光谱方法研究了不同环境气体压强和激光 功率密度的激光碳等离子体时空分辨光谱 ${ }^{[5]}$. 美国的 Filevich 等人 ${ }^{[6]}$ 利用类氖氩 $46.9 \mathrm{~nm}$ 软 $\mathrm{X}$ 射线激光作 为探针光, 通过干涉法获得了不同功率密度条件下 的 Nd:YAG 激光产生的 $\mathrm{Cu}$ 等离子体电子密度的时空 演化特征. 英国的 Hendron 等人 ${ }^{[7]}$ 利用朗缪尔探针技 术研究了 $\mathrm{Cu}$ 等离子体温度、电子密度和等离子体电 势随时间的演化情况. 另外还有很多研究小组利用 质谱法 ${ }^{[8,9]}$ 、Thomson 散射法 ${ }^{[10]}$ 等实验手段研究了激 光等离子体的动力学特性. 而在理论方面, 美国 Lawrence Livermore 国家实验室发展的 LASNEX 程 序, 不但能够较好地对激光惯性约束聚变实验进行 理论设计与分析, 还能研究激光等离子体辐射输运 性质 ${ }^{[11]}$. 北京应用物理与计算数学所的研究组发展 了平均原子 $(\mathrm{AA})$ 等理论模型 ${ }^{[12]}$, 进行了 LTE 和 NLTE 条件下等离子体辐射不透明度的研究 ${ }^{[13]}$. 另外, 该研究所的段斌等人 ${ }^{[14]}$ 基于对惯性约束聚变等离子 体特征发射光谱及其辐射线形的规律研究, 分析了 惯性约束聚变内爆实验的测量结果 ${ }^{[15]}$. 国防科技大 学的研究组发展了等离子体辐射不透明度的细致谱 项(DTA)模型, 对铝等元素的辐射不透明度进行了详 细的研究 ${ }^{[16,17]}$. 日本的 Hara 等人 ${ }^{[18]}$ 利用 STAR-2D 程 序 ${ }^{[19]}$ 研究了 $\mathrm{ps}$ 激光产生的微等离子体的辐射动力学 特性. 意大利的 Casavola 等人 ${ }^{[20]}$ 基于流体动力学方 法研究了非平衡状态下的 $\mathrm{Ti}$ 等离子体在不同初始条 件下的膨胀动力学过程. 该研究小组还在流体动力 学方程的基础上结合化学平衡模型, 研究了局域热 动平衡状态下的 $\mathrm{TiO}$ 等离子体的温度和密度的时间 演化 $^{[21]}$.

然而, 上述实验和理论研究主要涉及的是激光 与物质、激光与等离子体相互作用过程中的等离子体 动力学特性, 对处于后烧蚀过程(激光作用结束后)等
离子体性质的研究较少. 本文以激光烧蚀低 $Z$ 元素 $\mathrm{Si}$ 靶产生的等离子体时间-空间演化光谱为例, 建立了 一种简化的辐射动力学模型, 实现了等离子体时空 分辨光谱的理论模拟和演化图像的重构, 以期能够 使该模型运用于中高 $Z$ 等离子体中高电荷态离子辐 射特性的研究.

\section{1 实验方法和装置}

实验采用的时间-空间分辨激光等离子体发射光 谱测量装置如图 1 所示. 实验中, 使用 Nd:YAG 脉冲 激光器(PRO-350)发出的激光脉冲(波长 $1064 \mathrm{~nm}$, 脉 宽 $10 \mathrm{~ns}$ )经 $400 \mathrm{~mm}$ 的石英透镜聚焦在置于真空中的 高纯度 $\mathrm{Si}$ 靶(99.99\%)表面上产生等离子体. 平行于靶 面方向上的等离子体辐射光穿过宽度为 $10 \mu \mathrm{m}$ 的入 射狭缝进入掠入射极真空紫外光谱仪 (Mcpherson310G), 经 600 grooves $/ \mathrm{mm}$ 光栅分光后, 色散信号通 过直径为 $40 \mathrm{~mm}$ 微通道板耦合于 $1024 \times 255$ 像素的 面阵 $\mathrm{CCD}$ 探测器. 实验采用的光谱仪的分辨率为 $0.07 \mathrm{~nm}$.

为了避免激光脉冲在靶面同一位置重复烧蚀, 并实现等离子体的空间分辨测量, 样品靶被固定在 由三维电控平移系统控制的样品支架上, 而腔外的 石英聚焦透镜被固定于沿激光脉冲传播方向上的一 维电控平移系统台上. 相关运动路径和步长由 4 通道 电控平移台控制箱(MC600)程序控制, 最小移动步长 被设置为 $0.2 \mathrm{~mm}$. 同时, 为了实现光谱的时间分辨 测量, 两台数字脉冲延迟产生器(DG535 和 DG645)被 分别用于实现激光器、样品平移台控制箱、 $\mathrm{MCP}$ 和 $\mathrm{CCD}$ 探测器间的触发与时序控制. 外触发信号由脉 冲发生器 (8116A) 手动触发. 实验中, 真空腔室和光 谱仪中的真空度分别低于 $5.2 \times 10^{-4}$ 和 $4.6 \times 10^{-4} \mathrm{~Pa}$.

\section{2 理论模型}

为了从理论上获得激光等离子体在真空环境中 的膨胀速度、等离子体温度、离子密度等状态参量的 时间-空间演化信息, 本文在直角坐标系下建立了适 用于研究激光作用结束后( $>10 \mathrm{~ns})$ 的等离子体演化辐 射动力学模型.

考虑到激光等离子体的特征长度和特征时间均 远大于等离子体中粒子的平均自由程和平均碰撞时 间, 且自身整体呈电中性, 因此可以像流体力学中那 


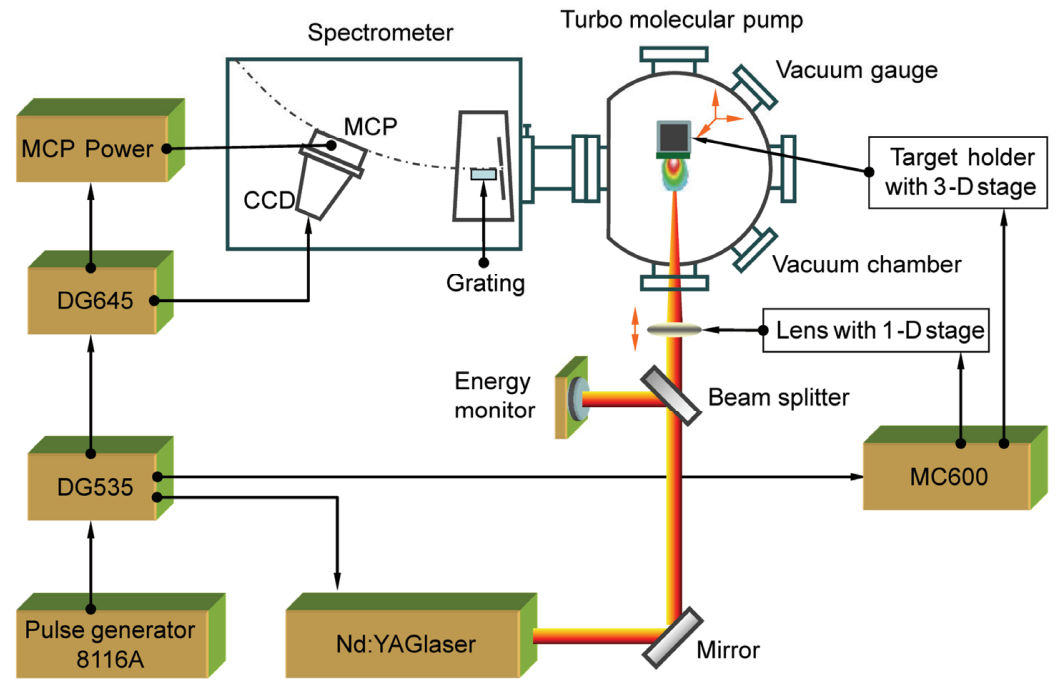

图 1 (网络版彩图)激光等离子体光谱实验装置图

Figure 1 (Color online) Schematic of the experimental setup used for laser produced plasma.

样定义等离子体的状态参量, 并用这些状态参量来 描述等离子体的宏观运动. 模型中涉及的流体力学 方程组由连续性方程、动量守恒方程、能量守恒方程 构成, 其形式如下 ${ }^{[22]}$ :

$$
\frac{\partial \rho}{\partial t}+\nabla \cdot(\rho \boldsymbol{u})=0
$$

$\rho \frac{\partial \boldsymbol{u}}{\partial t}+\rho(\boldsymbol{u} \cdot \nabla) \boldsymbol{u}=-\nabla p$

$\frac{\partial}{\partial t}\left(\rho \varepsilon+\rho u^{2} / 2\right)=-\nabla \cdot\left[\boldsymbol{u}\left(\rho \varepsilon+p+\rho u^{2} / 2\right)\right]-q$,

其中, $\rho$ 为等离子体密度,

$\rho=\left(m_{i} / x_{\mathrm{e}}+m_{\mathrm{e}}\right) n_{\mathrm{e}} \approx n m_{i}$,

$m_{i}$ 是等离子体粒子的平均质量, $n$ 为总的离子数密度, $u$ 为等离子体的膨胀速度,

$q=\int_{0}^{\infty} \mathrm{d} v \int \kappa_{v}\left(I_{v}^{b}-I_{v}\right) \mathrm{d} \Omega$

为辐射损失 ${ }^{[23]} . p$ 和 $\rho \varepsilon$ 分别为局部压强和局部内能 密度 ${ }^{[24]}$, 可由以下等离子体的状态方程确定:

$p=\left(1+x_{\mathrm{e}}\right) n k T$,

$\rho \varepsilon=n\left[\frac{3}{2}\left(1+x_{\mathrm{e}}\right) k T+\sum_{\omega=1}^{i_{\max }}\left(E_{\omega} \sum_{i=1}^{i_{\max }} x_{i}\right)\right]$,

式中, $k T$ 和 $n_{\mathrm{e}}$ 分别为等离子体温度和电子密度, $x_{\mathrm{e}}=$ $n_{\mathrm{e}} / n$ 为平均离化度, $x_{i}=n_{i} / n$ 为 $i$ 次离化的离子丰度, $n_{i}$ 为 $i$ 电离度离子密度, $E_{\omega}$ 为剥离 $\omega$ 个电子所需的最 小能量.

在高功率纳秒激光作用于靶面产生的等离子体 中，粒子间的碰撞非常频繁，系统的平衡在非常短的 时间内 $(\sim 10 \mathrm{ps})$ 就能达到, 可以认为等离子体处于局 域的热力学平衡状态, 这意味着在等离子体中的每 一空间点电子与离子以及其他粒子的温度几乎相同. 因此理论模型中的原子、离子和电子数应遵从 Saha 方程 ${ }^{[23]}$, 即

$\frac{n_{i+1} n_{\mathrm{e}}}{n_{i}}=2\left(\frac{2 \pi m k T}{h^{2}}\right)^{3 / 2} \frac{U_{i+1}}{U_{i}} \mathrm{e}^{-\left(\phi_{i+1}-\Delta \phi_{i+1}\right) / k T}$,

式中, $U_{i}$ 为 $i$ 电离度离子的配分函数, $\phi_{i+1}$ 为电离能, $\Delta \phi_{i+1}=3 \times 10^{-8} \mathrm{Zn}_{\mathrm{e}}^{1 / 2} T^{-1 / 2} \mathrm{eV}$ 表示等离子体效应导致的 电离能下降. 结合离子数守恒方程 $n=\sum_{i=0}^{Z} n_{i}$ 和电荷 守恒方程 $n_{\mathrm{e}}=\sum_{i=0}^{Z} i n_{i}$, 可以进行电子、离子数密度 以及平均离化度等物理量的求解.

激光等离子体是一种既发射辐射同时也吸收辐 射的等离子体介质, 其辐射强度的变化规律可以用 辐射输运方程来表示, 具体形式为 ${ }^{[25]}$

$\Omega \operatorname{grad} I_{v}+\kappa_{v} I_{v}=\kappa_{v} I_{v}^{b}$,

式中, $I_{v}$ 以及 $I_{v}^{b}$ 分别为等离子体辐射强度和黑体辐 射强度. $\kappa_{v}$ 为有效吸收系数, 可以表示成 $\kappa_{v}=\kappa_{\mathrm{ff}}+\kappa_{\mathrm{fb}}+\kappa_{\mathrm{bb}}$, 
其中 $\kappa_{\mathrm{ff}}$ 为自由-自由有效吸收系数、 $\kappa_{\mathrm{fb}}$ 为束缚-自由 有效吸收系数、 $\kappa_{\mathrm{bb}}$ 为束缚-束缚有效吸收系数 ${ }^{[23]}$. 其 具体形式为

$$
\begin{aligned}
& \kappa_{\mathrm{ff}}=\frac{8 \pi \mathrm{e}^{6}}{3 m_{\mathrm{e}} h c\left(6 \pi m_{\mathrm{e}} k\right)^{1 / 2}} \frac{n_{\mathrm{e}} G}{T^{1 / 2} v^{3}}\left(\mathrm{e}^{h v / k T}-1\right) \sum_{i} i^{2} n_{i}, \\
& \kappa_{\mathrm{fb}}=\frac{8 \pi \mathrm{e}^{6}}{3 m_{\mathrm{e}} h c\left(6 \pi m_{\mathrm{e}} k\right)^{1 / 2}} \frac{n_{\mathrm{e}} \xi_{z} \mathrm{e}^{h v / k T}}{T^{1 / 2} v^{3}}\left(1-\mathrm{e}^{-h v / k T}\right)^{2} \sum_{i} i^{2} n_{i}, \\
& \kappa_{\mathrm{bb}}=\left(1-\mathrm{e}^{-h v / k T}\right) \frac{h v}{c} \sum_{i} \sum_{l, u} B_{l u} n_{i l} P_{l u} .
\end{aligned}
$$

在上述公式中, $n_{\mathrm{e}}$ 和 $n_{i}$ 分别为电子和离子数密度, $G$ 是冈特因子, $B_{l u}$ 为电离度为 $i$ 的离子从能态 $l$ 到 $u$ 的爱因斯坦系数, $n_{i l}$ 表示电离度为 $i$ 的离子的 $l$ 能态的 粒子数密度, $P_{l u}$ 为光谱线型.

由于本文建立的激光等离子体辐射动力学模型, 主要研究的是激光作用结束后等离子体的特性. 因 此, 初始条件和边界条件的选择极为重要. 我们将激 光作用刚结束的时刻取为时间零点. 根据 Harilal 等

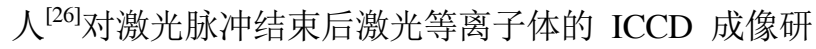
究, 其形状可近似为沿激光入射方向的轴对称椭球, 在 平行于靶面方向的尺寸近似为激光焦斑直径 $Y(0) \approx 0.04 \mathrm{~cm}$, 垂直于靶面方向的尺寸近似为 $X(0) \approx 0.01 \times\left(T_{0} / A\right)^{1 / 2} \mathrm{~cm}(A \text { 为相对原子质量 })^{[27]}$. 同时假 定初始等离子体的温度和离子密度满足高斯分布 ${ }^{[28,29]}$

$$
\begin{aligned}
& T(x, y, 0)=T_{0} \exp \left(-\frac{x^{2}}{2 X(0)^{2}}-\frac{y^{2}}{2 Y(0)^{2}}\right), \\
& n(x, y, 0)=n_{0} \exp \left(-\frac{x^{2}}{2 X(0)^{2}}-\frac{y^{2}}{2 Y(0)^{2}}\right),
\end{aligned}
$$

其中 $T_{0}$ 为等离子体中心处的温度, 可以由 $T_{0}=$ $5.2 \times 10^{-6} Z^{1 / 5}\left(\lambda^{2} \Phi\right)^{3 / 5}{ }^{[30]}$ 确定. 其中, $\lambda$ 为激光波长,

$\Phi$ 为激光功率密度.

另外, 由于等离子体在真空中膨胀, 所以边界处 的压强 $p=0$.

\section{3 结果与讨论}

\section{1 激光 $\mathrm{Si}$ 等离子体时间-空间分辨光谱测量及分 析模拟}

激光等离子体内在的瞬态演变和不均匀特性要 求在光谱测量时必须进行高分辨的时间-空间演化测
量. 为了能够准确地揭示等离子体的时间-空间演化 特性, 我们细致测量了激光功率密度为 $3.0 \times 10^{11}$ $\mathrm{W} / \mathrm{cm}^{2}$ 时的不同延迟时间和不同空间探测位置的 $\mathrm{Si}$ 等离子体发射光谱, 波长范围从 7.0-13.0 nm. 图 2(a) 和(b)分别给出了空间探测位置为 $1.5 \mathrm{~mm}$ 处的时间分 辨光谱和延迟时间为 $40 \mathrm{~ns}$ 的空间分辨光谱. 从图 2(a) 可以清楚看出, 随着时间延迟的增加谱线强度逐渐 增强，在延迟时间为 $45 \mathrm{~ns}$ 时谱线强度达到最强，随 后逐步减弱, 直至 $90 \mathrm{~ns}$ 后完全消失. 而在图 2(b)中, 距靶面 $1.5 \mathrm{~mm}$ 处光谱强度达到最强, 在远离靶面 3 $\mathrm{mm}$ 处谱线消失. 同时, 也可以看出在真空环境中, 靶面附近和延迟时间较短时等离子体的连续辐射非 常弱，接近于背景强度，其光谱特征与大气压空气环
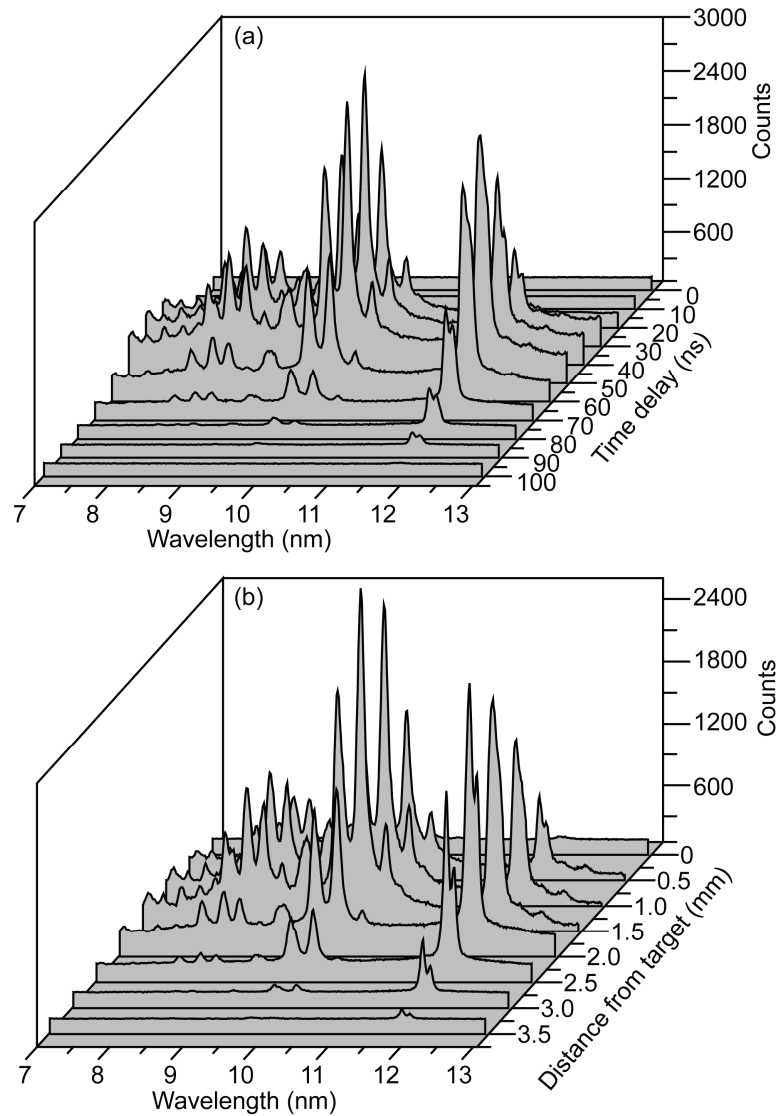

图 2 激光烧蚀产生的 $\mathrm{Si}$ 等离子体时间-空间分辨光谱. (a) 空间探测位置为 $1.5 \mathrm{~mm}$ 处的时间分辨光谱; (b) 延迟时间为 $40 \mathrm{~ns}$ 的空间分辨光谱

Figure 2 Time-space resolved spectra of laser produced Si plasma. (a) Time resolved spectra of distance from target $1.5 \mathrm{~mm}$; (b) space resolved spectra of time delay $40 \mathrm{~ns}$. 
境的激光诱导击穿光谱特征截然不同 ${ }^{[31]}$.

为了辨认光谱轮廓中分立谱线的来源, 以延迟

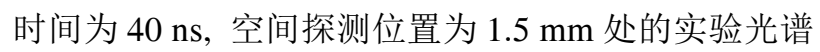
为例, 对分立光谱轮廓进行了 Lorentz 线型拟合, 获 得了谱线中心波长、半高全宽等谱线信息. 通过与 NIST 数据库中 $\mathrm{Si}$ 原子参数的对比分析, 确定了实验 光谱中的分立谱峰主要源于 $\mathrm{Si}^{4+}-\mathrm{Si}^{6+}$ 离子的 $2 \mathrm{p}-3 \mathrm{~s}$, $3 \mathrm{~d}$ 跃迁, 如图 3 所示. 另外, 为了便于进行光谱模拟 和等离子体参数诊断, 表 1 给出了相应的原子结构 参数.

从图 3 可以看出, 较强的谱线主要来自于 $\mathrm{Si} \mathrm{VI}$ 和 $\mathrm{V}$ 离子的 $2 \mathrm{p}-3 \mathrm{~s}$ 跃迁, 这些谱线主要分布于光谱区 域的中部和长波区域. 而源于 $\mathrm{Si}$ VI 和 VII 离子的 $2 \mathrm{p}-3 \mathrm{~d}$ 跃迁主要分布在短波区域, 且强度较弱.

为了能够准确地揭示等离子体的演化特性，同 时验证初始条件选择的合理性, 本文利用建立的辐 射动力学模型对图 2 中的时间-空间分辨光谱进行理 论模拟, 归一化的实验和理论模拟光谱对比结果如 图 4 所示. 其中实验光谱以灰色阴影区域表示, 理论 模拟结果以黑色加粗实线表示. 从图中可以明显看 出, 理论模拟光谱与实验测量光谱符合较好, 表明本 文建立的辐射动力学模型能够准确地重构实验光谱 和等离子体的演化过程, 选取的初始条件可以较好

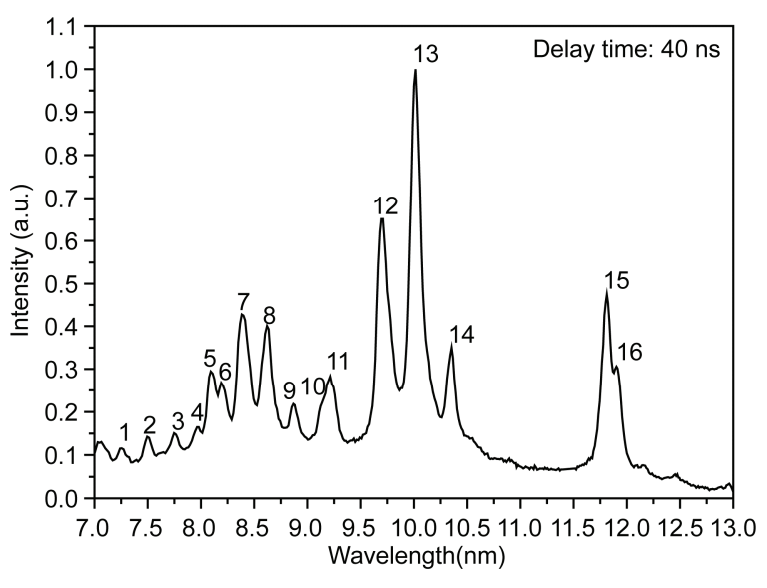

图 3 激光产生的 $\mathrm{Si}$ 等离子体光谱

Figure 3 Spectra of laser produced Si plasma.

地反映模型初始时刻等离子体的状态.

\section{2 等离子体状态参数随时间和空间的演化}

为了进一步揭示等离子体的膨胀和冷却过程, 基于对上述的实验和理论模拟结果的比较, 图 5 给出 了垂直于靶面方向的等离子体边缘速度和尺寸随时 间的演化曲线. 从图中可以看出, 初始阶段等离子体 的膨胀速度增加很快, 随后逐渐变缓. 表明在激光脉 冲作用后几十纳秒内, 随着等离子体体积的膨胀, 由

表 $1 \mathrm{Si}^{4+}-\mathrm{Si}^{6+}$ 离子的谱线跃迁信息

Table 1 Transition wavelength and weighted transition probabilities for $\mathrm{Si}^{4+}-\mathrm{S}^{6+}$ ions

\begin{tabular}{|c|c|c|c|c|c|c|c|}
\hline \multirow{2}{*}{ Index } & \multicolumn{2}{|c|}{ Wavelength(nm) } & \multirow[b]{2}{*}{ Ion } & \multicolumn{4}{|c|}{ Transition } \\
\hline & Experiment & NIST & & Configuration & Term & $\mathrm{J}-\mathrm{J}$ & $\mathrm{gA}_{\mathrm{ki}}\left(\mathrm{s}^{-1}\right)$ \\
\hline 1 & 7.256 & 7.249 & $\mathrm{Si}^{6+}$ & $2 s^{2} 2 p^{4}-2 s^{2} 2 p^{3} 3 d$ & ${ }^{1} \mathrm{D}-{ }^{-3} \mathrm{~F}$ & $2-2$ & $9.35 \times 10^{9}$ \\
\hline 2 & 7.496 & 7.519 & $\mathrm{Si}^{6+}$ & $2 s^{2} 2 p^{4}-2 s^{2} 2 p^{3} 3 d$ & ${ }^{1} \mathrm{~S}-{ }^{1} \mathrm{P}$ & $0-1$ & $9.30 \times 10^{10}$ \\
\hline 3 & 7.748 & 7.741 & $\mathrm{Si}^{5+}$ & $2 s^{2} 2 p^{5}-2 s^{2} 2 p^{4} 3 d$ & ${ }^{2} \mathrm{P}-{ }^{2} \mathrm{D}$ & $3 / 2-3 / 2$ & $3.10 \times 10^{10}$ \\
\hline 4 & 7.980 & 7.962 & $\mathrm{Si}^{6+}$ & $2 s^{2} 2 p^{4}-2 s^{2} 2 p^{3} 3 s$ & ${ }^{3} \mathrm{P}-{ }^{3} \mathrm{P}$ & $0-1$ & $5.52 \times 10^{10}$ \\
\hline 5 & 8.094 & 8.090 & $\mathrm{Si}^{6+}$ & $2 s^{2} 2 p^{4}-2 s^{2} 2 p^{3} 3 s$ & ${ }^{3} \mathrm{P}-{ }^{1} \mathrm{D}$ & $2-2$ & $6.15 \times 10^{9}$ \\
\hline 6 & 8.191 & 8.189 & $\mathrm{Si}^{6+}$ & $2 s^{2} 2 p^{4}-2 s^{2} 2 p^{3} 3 s$ & ${ }^{3} \mathrm{P}-{ }^{3} \mathrm{D}$ & $1-1$ & $6.21 \times 10^{10}$ \\
\hline 7 & 8.384 & 8.363 & $\mathrm{Si}^{5+}$ & $2 s^{2} 2 p^{5}-2 s^{2} 2 p^{4} 3 d$ & ${ }^{2} \mathrm{P}-{ }^{2} \mathrm{P}$ & $1 / 2-1 / 2$ & $8.72 \times 10^{10}$ \\
\hline 8 & 8.610 & 8.570 & $\mathrm{Si}^{6+}$ & $2 s^{2} 2 p^{4}-2 s^{2} 2 p^{3} 3 s$ & ${ }^{3} \mathrm{P}-{ }^{3} \mathrm{~S}$ & $0-1$ & $4.62 \times 10^{10}$ \\
\hline 9 & 8.867 & 8.883 & $\mathrm{Si}^{6+}$ & $2 s^{2} 2 p^{4}-2 s^{2} 2 p^{3} 3 s$ & ${ }^{1} \mathrm{~S}-{ }^{3} \mathrm{D}$ & $0-1$ & $2.39 \times 10^{8}$ \\
\hline 10 & 9.125 & 9.137 & $\mathrm{Si}^{5+}$ & $2 s^{2} 2 p^{5}-2 s^{2} 2 p^{4} 3 s$ & ${ }^{2} \mathrm{P}-{ }^{2} \mathrm{~S}$ & $3 / 2-1 / 2$ & $3.92 \times 10^{10}$ \\
\hline 11 & 9.204 & 9.180 & $\mathrm{Si}^{5+}$ & $2 s^{2} 2 p^{5}-2 s^{2} 2 p^{4} 3 s$ & ${ }^{2} \mathrm{P}-{ }^{2} \mathrm{~S}$ & $1 / 2-1 / 2$ & $2.46 \times 10^{10}$ \\
\hline 12 & 9.688 & 9.649 & $\mathrm{Si}^{5+}$ & $2 s^{2} 2 p^{5}-2 s^{2} 2 p^{4} 3 s$ & ${ }^{2} \mathrm{P}-{ }^{2} \mathrm{D}$ & $1 / 2-3 / 2$ & $1.12 \times 10^{11}$ \\
\hline 13 & 10.005 & 9.946 & $\mathrm{Si}^{5+}$ & $2 s^{2} 2 p^{5}-2 s^{2} 2 p^{4} 3 s$ & ${ }^{2} \mathrm{P}-{ }^{2} \mathrm{P}$ & $3 / 2-3 / 2$ & $2.70 \times 10^{11}$ \\
\hline 14 & 10.352 & 10.316 & $\mathrm{Si}^{5+}$ & $2 s 2 p^{6}-2 s 2 p^{5} 3 s$ & ${ }^{2} \mathrm{~S}-{ }^{2} \mathrm{P}$ & $1 / 2-3 / 2$ & $1.15 \times 10^{11}$ \\
\hline 15 & 11.815 & 11.786 & $\mathrm{Si}^{4+}$ & $2 s^{2} 2 p^{6}-2 s^{2} 2 p^{5} 3 s$ & ${ }^{1} \mathrm{~S}-{ }^{1} \mathrm{P}$ & $0-1$ & $1.07 \times 10^{11}$ \\
\hline 16 & 11.923 & 11.897 & $\mathrm{Si}^{4+}$ & $2 s^{2} 2 p^{6}-2 s^{2} 2 p^{5} 3 s$ & ${ }^{1} \mathrm{~S}-{ }^{3} \mathrm{P}$ & $0-1$ & $1.15 \times 10^{10}$ \\
\hline
\end{tabular}



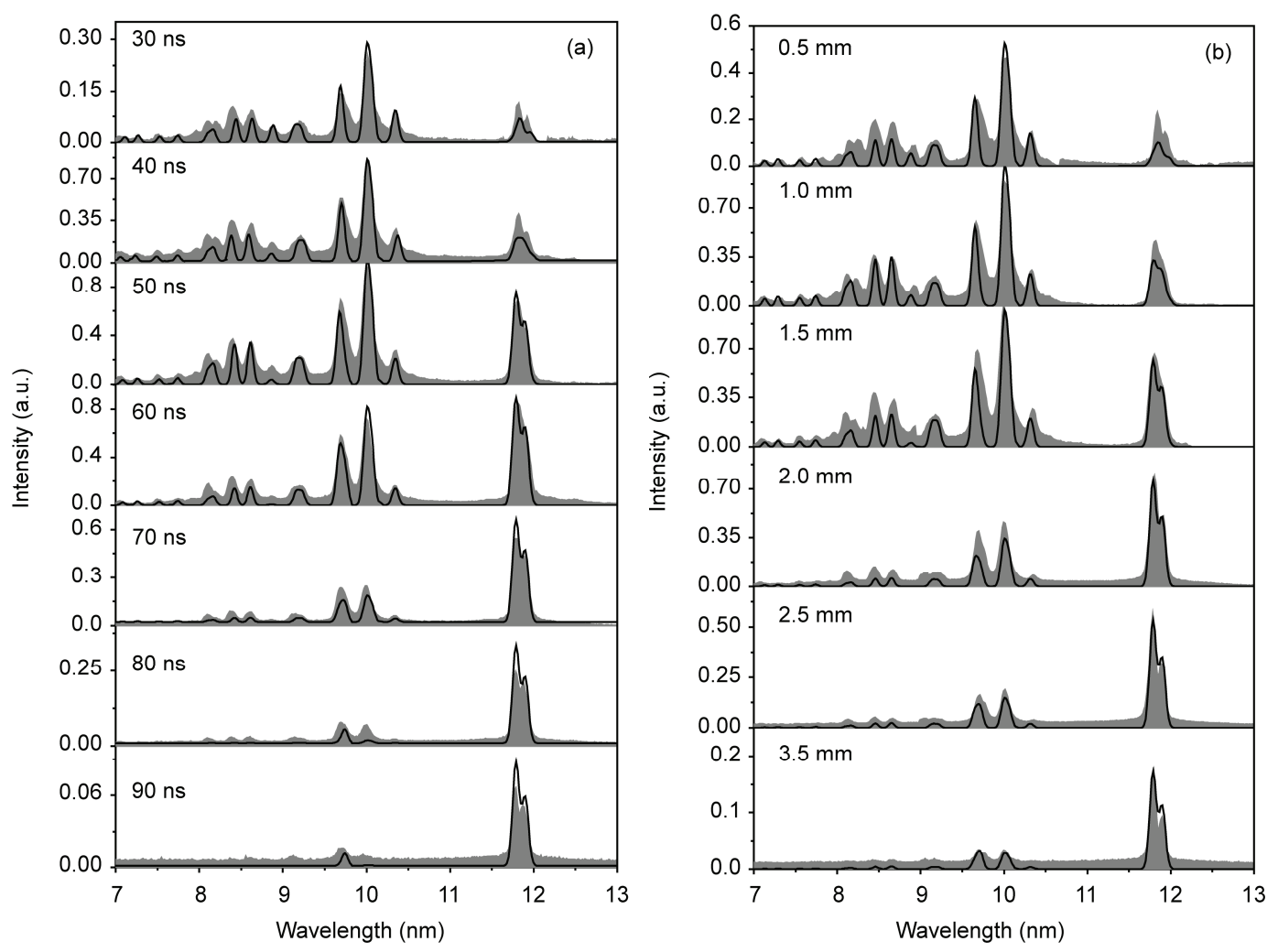

图 $4 \mathrm{Si}$ 等离子体归一化的时间-空间分辨光谱. (a) 探测位置距离靶面 $1.5 \mathrm{~mm}$ 的时间分辨光谱图; (b) 延迟时间 $40 \mathrm{~ns}$ 的空间 分辨光谱图. 图中灰色阴影区域表示实验光谱, 黑色加粗实线表示理论模拟结果

Figure 4 Experimental and theoretical spectra of Si plasma. (a) Distance from target of $1.5 \mathrm{~mm}$; (b) delay time of $40 \mathrm{~ns}$. The dark grey shaded area on this graph is experimental spectra, the black solid line in bold is theory simulation spectra.

于不存在激光能量的继续作用和来自靶面的烧蚀粒 子产生, 等离子体内能很快转化为动能, 使得等离子 体温度迅速降低, 平均自由程增大, 导致粒子间碰撞 的次数降低. 因此这一阶段的等离子体边缘的加速 度会快速降低, 但速度仍然是增加的.

等离子体温度和电子数密度是等离子体中两个 最重要的参数, 与等离子体辐射过程密切相关. 为直 观起见, 图6 给出了 $\mathrm{Si}$ 等离子体羽在真空中膨胀的时 间演化图像, 以及相应的等离子体温度和电子密度 的演化轮廓. 图中颜色梯度代表等离子体温度的变 化, 等高线代表电子密度. 结合图 5 和 6 可以清楚地 看出, 随着延迟时间的增加, 等离子体的体积逐渐增 大, 相应的等离子体温度从近靶面处 $30 \mathrm{eV}$ 快速衰变 到 $24 \mathrm{eV}$, 电子密度也有明显的梯度变化. 同时表明, 在高功率密度激光产生的等离子体中由于高电荷态 离子的存在, 其碰撞过程和复合过程随时间和空间 的变化非常剧烈. 从等离子体核心到边缘存在着明
显的等离子体温度梯度和密度梯度, 也证实等离子 体是高度不均匀的和瞬变的.

为了进一步描述等离子体中高电荷态离子的分 布, 图 7 给出了延迟时间为 $40 \mathrm{~ns}$ 的等离子体中总离 子数密度, 以及 Si IV-VIII 离子数密度的空间分布图 像. 从图中可以清晰看出在该时刻不同电荷态离子的 分布区域, 其中等离子体核心处主要为 Si VIII 和 VII 离子, 中间区域主要为 $\mathrm{Si} V \mathrm{VI}$ 和 $\mathrm{V}$ 离子, 最边缘的为 $\mathrm{Si}$ IV. 这种空间分布也进一步解释了图 6 的演化结果.

\section{4 结论}

本文基于对激光产生的 $\mathrm{Si}$ 等离子体 $\mathrm{EUV}$ 波段的 时间-空间分辨光谱的实验测量, 结合流体力学方程 与辐射输运方程建立了一种简化的辐射动力学模型, 系统分析模拟了激光产生的 $\mathrm{Si}$ 等离子体的时间-空间 分辨光谱, 获得了 $\mathrm{Si}$ 等离子体在真空膨胀时的速度、 


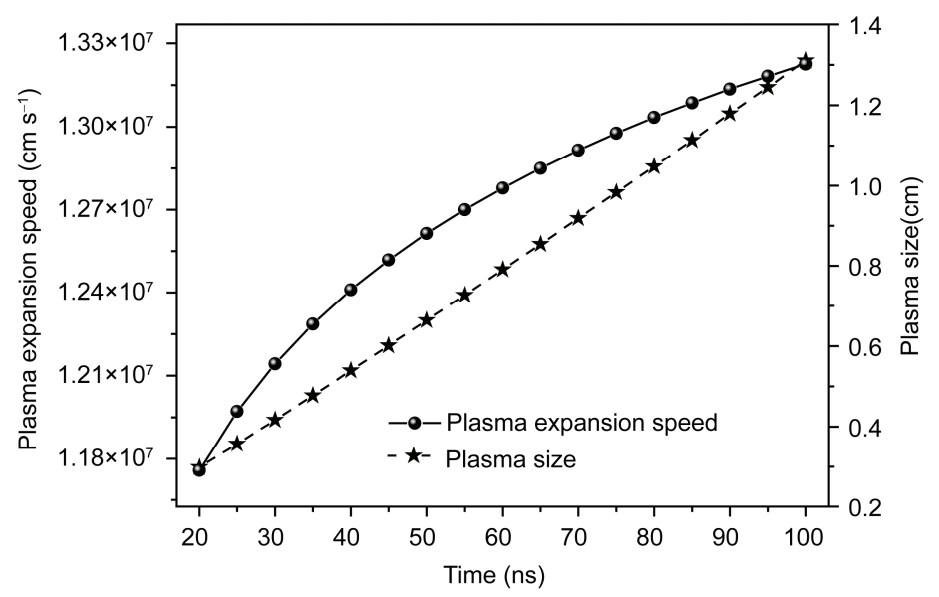

图 5 垂直于靶面方向的 $\mathrm{Si}$ 等离子体边缘速度及尺寸随时间的演化

Figure 5 Velocity and size evolution of Si plasma periphery in direction perpendicular to target surface with time.

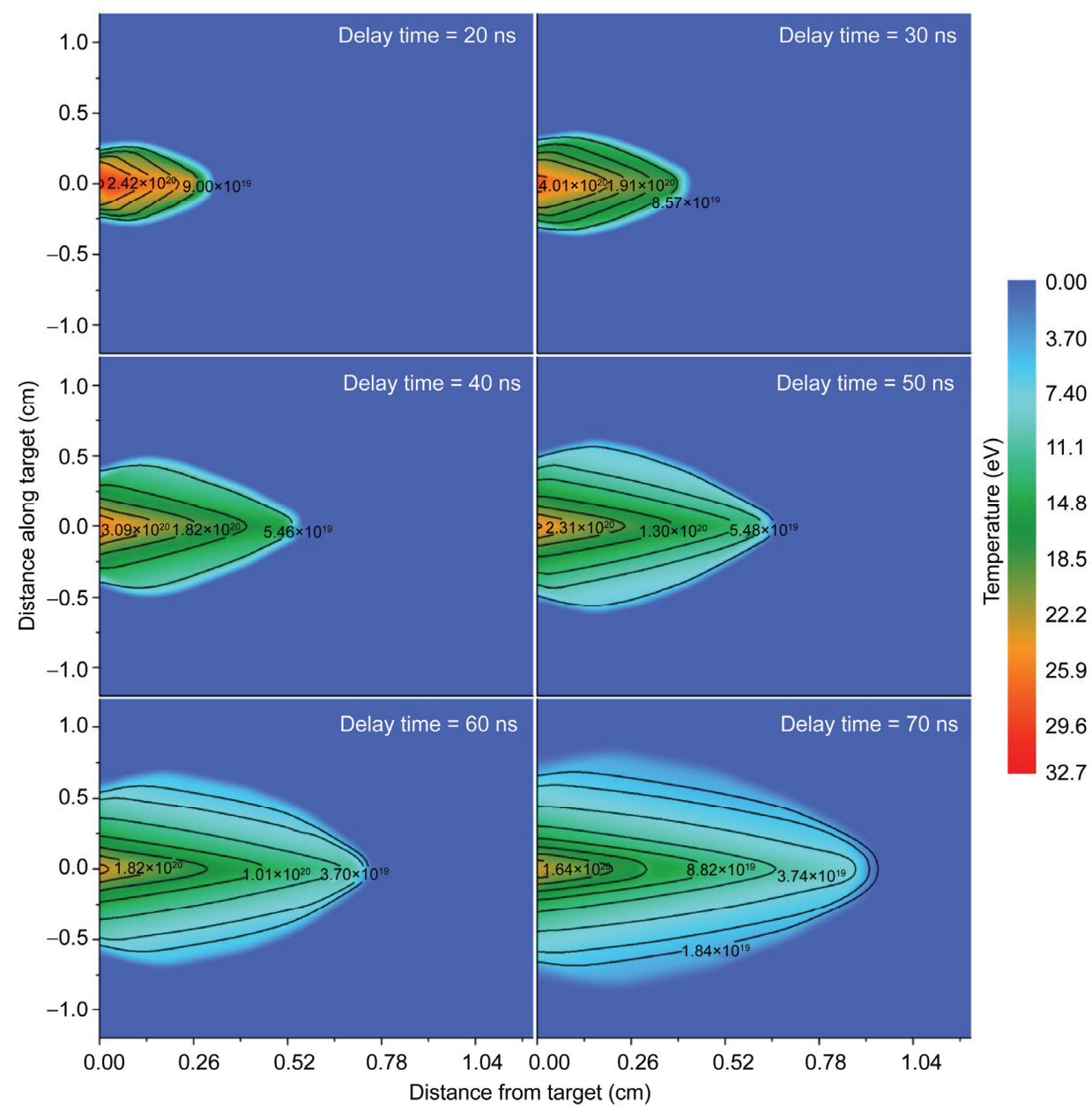

图 6 (网络版彩图) Si 等离子体温度与电子密度的时空演化图像

Figure 6 (Color online) Temporal-spatial evaluation of plasma temperature (filled contour) and electron density (line contours) in laser produced $\mathrm{Si}$ plasma. 


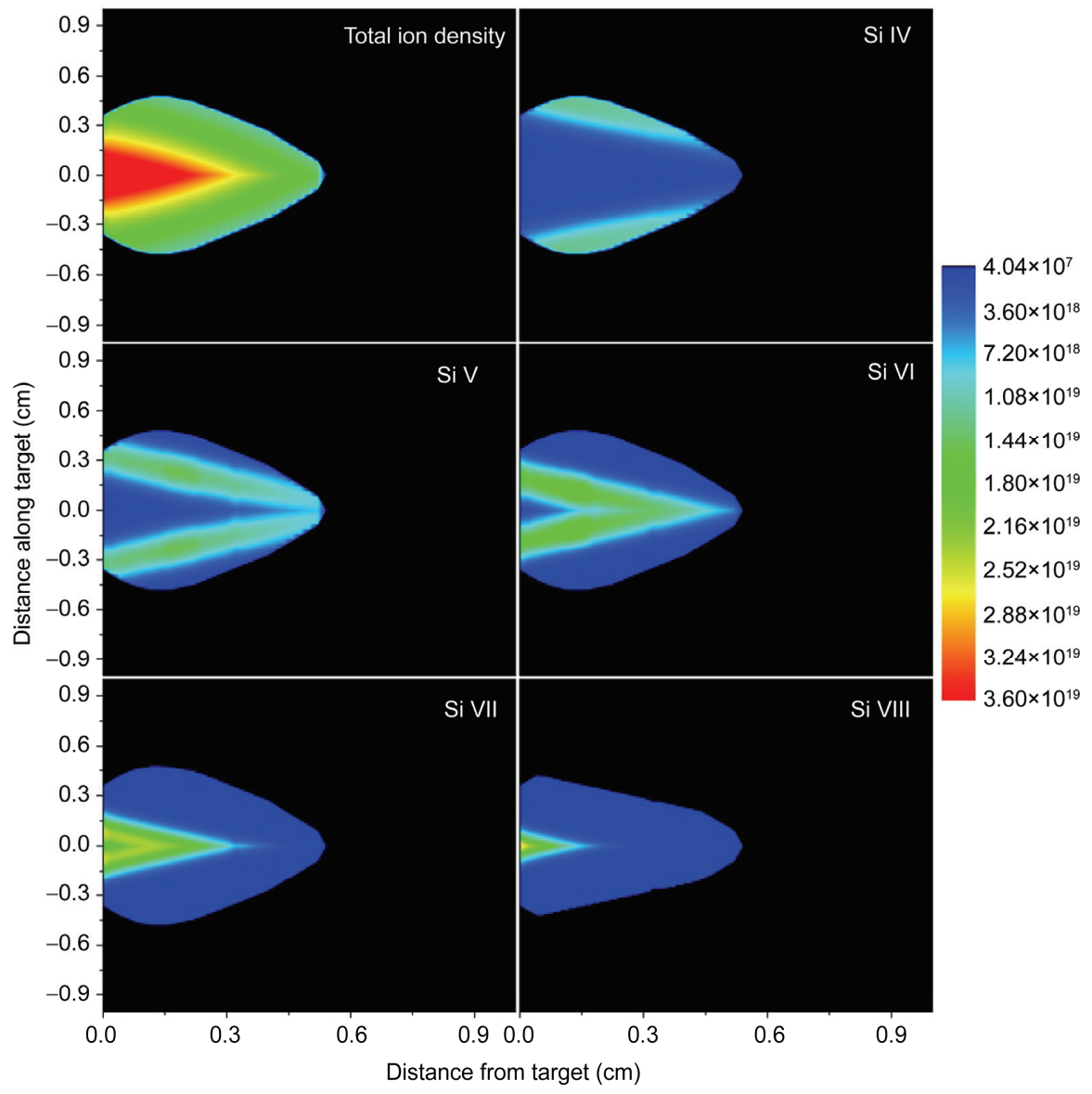

图 7 (网络版彩图) Si 等离子体中各种粒子数密度的时空演化图像

Figure 7 (Color online) Temporal-spatial evaluation of ion density (filled contour) in laser produced Si plasma.

等离子体温度、电子密度等状态参量随时间-空间的 演化图像，准确地再现了等离子体的膨胀和冷却过
程, 实现了等离子体的快速诊断, 为激光产生的高电 荷态离子辐射特性的研究提供了一种有效的方法.

\section{参考文献}

1 Carroll P K, Kennedy E T. Laser-produced plasmas. Contemp Phys, 1981, 22: 61-96

2 Glenzer S H, Fournier K B, Wilson B G, et al. Ionization balance in inertial confinement fusion hohlraums. Phys Rev Lett, 2001, 87: 045002

3 Xu Y, Zhang J Y, Yang J M, et al. A clean radiation environment for opacity measurements of radiatively heated material. Phys Plasmas, 2007, 14: 05270

4 Harilal S S, Bindhu C V, Tillack M S, et al. Internal structure and expansion dynamics of laser ablation plumes into ambient gases. J Appl Phys, 2003, 93: 2380-2388

5 Harilal S S. Expansion dynamics of laser ablated carbon plasma plume in helium ambient. Appl Surf Sci, 2001, 172: 103-109

6 Filevich J, Rocca J J, Jankowska E, et al. Two-dimensional effects in laser-created plasmas measured with soft-x-ray laser interferometry. Phys Rev E, 2003, 67: 056409

7 Hendron J M, Mahony C M O, Morrow T, et al. Langmuir probe measurements of plasma parameters in the late stages of a laser ablated plume. Appl Phys, 1997, 81: 2131-2134 
8 Park S M, Moon J Y. Laser ablation of graphite in an oxygen jet. J Chem Phys, 1998, 109: 8124-8129

9 Velotta R, Berardi V, Spinelli N, et al. Kinetic-energy distributions of charged fragments from $\mathrm{CO}_{2}$ dissociative ionization. J Phys B-At Mol Opt Phys, 1994, 27: 2051-2061

10 Warner K, Hieftje G M. Thomson scattering from analytical plasmas. Spectrochim Acta Part B, 2002, 57: 201-241

11 Estabrook K. LLNL Nova laser irradiated gold disk experiments diagnosed with Thomson scattering compared to LASNEX simulations. Livermore: Lawrence Livermore National Laboratory, 1998. UCRL-JC-130984

12 Pei W B, Chang T Q. Improved calculation for ion configuration distribution from the average-atom model. J Quant Spectrosc Ra, 2000, 64: $15-23$

13 Yuan J K, Sun Y S, Zheng S T. Calculation of the electrical conductivity of strongly coupled plasmas. Phys Rev E, 1996, 53: 1059-1067

14 Duan B, Wu Z Q, Wang J G. Spectral diagnostics of inertial confinement fusion plasma (I). Sci China Ser G-Phys Mech Astron, 2009, 39: 43-51 [段斌，吴泽清，王建国. 惯性约束聚变等离子体的光谱诊断(I). 中国科学 $\mathrm{G}$ 辑: 物理学 力学 天文学, 2009, 39: 43-51]

15 Duan B, Wu Z Q, Wang J G. Spectral diagnostics of inertial confinement fusion plasma (II). Sci China Ser G-Phys Mech Astron, 2009, 39: 241-248 [段斌, 吴泽清, 王建国. 惯性约束聚变等离子体的光谱诊断(II). 中国科学 G 辑: 物理学 力学 天文学, 2009, 39: 241-248]

16 Zeng J L, Jin F T, Yuan J M, et al. Detailed-term-accounting-approximation simulation of X-ray transmission through laser-produced Al plasmas. Phys Rev E, 2000, 62: 7251-7257

17 Zeng J L, Yuna J M, Lu Q S. Detailed-term-accounting-approximation calculations of the radiative opacity of laser-produced Al plasmas. Phys Rev E, 2001, 64: 066412

18 Hara H, Arai G, Dinh T H, et al. Numerical evaluation of a $13.5 \mathrm{~nm}$ high-brightness microplasma extreme ultraviolet source. J Appl Phys, 2015, 118: 193301

19 Sunahara A, Johzaki T, Nagatomo H, et al. Generation of pre-formed plasma and its reduction for fast-ignition. Laser Part Beams, 2012, 30: 95-102

20 Casavola A, Colonna G, Capitelli M. Non-equilibrium conditions during a laser induced plasma expansion. Appl Surf Sci, 2003, 208-209: 85-89

21 Colonna G, Casavola A, Capitelli M. Modeling of LIBS plasma expansion. Spectrochim Acta Part B, 2001, 56: 567-586

22 Zel'dovich Y B, Raizer Y P. Physics of Shock Waves and High-Temperature Hydrodynamic Phenomena. New York: Academic Press, 1966

23 Gornushkin I B, Kazakov A Y, Omenetto N, et al. Radiation dynamics of post-breakdown laser induced plasma. Spectroc Acta Pt B, 2004, 59: 401-418

24 Shabanov S V, Gornushkin I B. Two-dimensional axisymmetric models of laser induced plasmas relevant to laser induced breakdown spectroscopy. Spectroc Acta Pt B, 2014, 100: 147-172

25 Mazhukin V I, Nossov V V, Flamant G, et al. Modeling of radiation transfer and emission spectra in laser-induced plasma of Al vapor. J Quant Spectrosc Ra, 2002, 73: 451-460

26 Harilal S S. Influence of spot size on propagation dynamics of laser-produced tin plasma. J Appl Phys, 2007, 102: 123306

27 Anisimov S I, Bauerle D. Gas dynamics and film profiles in pulsed-laser deposition of materials. Phys Rev B, 1993, 48: 12076-12081

28 Singh R K, Narayan J. Pulsed-laser evaporation technique for deposition of thin films: Physics and theoretical model. Phys Rev B, 1990, 41: 8843-8859

29 Pakhal H R, Lucht R P, Laurendeau N M. Spectral measurements of incipient plasma temperature and electron number density during laser ablation of aluminum in air. Appl Phys B, 2008, 90: 15-27

30 Colombant D, Tonon G F. X-ray emission in laser-produced plasmas. J Appl Phys, 1973, 44: 3524-3537

31 Capitelli M, Casavola A, Colonna G. Laser-induced plasma expansion: Theoretical and experimental aspects. Spectroc Acta Pt B, 2004, 59: 271-289 


\title{
Investigation of radiation dynamic properties in laser-produced silicon plasma
}

\author{
MIN Qi, SU MaoGen*, CAO ShiQuan, SUN DuiXiong \& DONG ChenZhong* \\ Key Laboratory of Atomic and Molecular Physics \& Functional Material of Gansu Province, College of Physics and Electronic \\ Engineering, Northwest Normal University, Lanzhou 730070, China
}

In this paper, the radiation characteristic of laser produced plasma in vacuum environment is studied from experiment and theory. Experimentally, the EUV emission spectrum of the Si plasma is measured by using the time-space resolved laser-produced plasma technique, and the spectrum is identified and analyzed. In order to explore the dynamic properties of the experimental spectra, a simplified radiation dynamic model, which can be used to investigate the radiation properties and dynamics evolution of the laser-produced plasma is established on the basis of the gas dynamic equations and the radiative transfer equation. By using this model, the time-space resolved spectrum is simulated and the temporal and spatial evolution characteristics of the plasma temperature and density as well as the various transient ions are investigated systematically. The results show that the presented radiation dynamic model can reflect the dynamical evolution process of the laser produced plasma and radiation characteristics of the emission spectra very well, and therefore it can be used as an effective tool in laser produced plasma diagnosis.

laser-produced Si plasma, time-space resolved spectra, radiation dynamic model

PACS: $32.30 . J c, 39.30 .+w, 47.10 . a b, 57.70 . K z$

doi: 10.1360/SSPMA2016-00032 